\title{
DRUG INDUCED ACUTE TUBULAR NECROSIS - RARE CASE OF NEPHROTIC SYNDROME
}

\author{
Magdalena Starcea ${ }^{1,2}$, Mihaela Munteanu², Radu Russu ${ }^{2}$, Anca Iulia Rotaru', \\ Doina Mihaila ${ }^{3}$, Ingrith Miron ${ }^{1,2}$ \\ I "Gr. T. Popa" University of Medicine and Pharmacy, Iasi \\ ${ }^{2}$ Department of Pediatric Nephrology, \\ "Sf. Maria” Emergency Clinical Hospital for Children, Iasi \\ ${ }^{3}$ Laboratory of Pathology, "Sf. Maria” Emergency Clinical Hospital for Children, Iasi
}

\begin{abstract}
We presented two cases of nephrotic syndrome (NS) drug-induced with tubular nephrotoxicity, with different evolution in the context of etiologic diseases.

First is 5-month-old girl admitted with NS (clinically and biological proven) and acute renal failure after another hospitalization for pneumonia. The girl was treated with ceftriaxone and gentamicin 12 days. Congenital NS suspicion was eliminated by renal biopsy who revealed renal tubular necrosis highlighting recovery phase. The development was favorable in 7 days of peritoneal dialysis.

The second case was 16 years old adolescents treated 3 years with carbimazol for Basedow disease. Was presented with nephrotic syndrome not influenced by corticosteroids. Histopathology revealed toxic tubular necrosis, interstitial fibrosis, absence of glomerular injury. Nephrotoxic treatment was stopped, and, after thyroidectomy, edema were reduced, but kidney function continued to depreciate, while nephrotoxic therapy given for 3 years.

Conclusions. Renal tubular necrosis clinical and laboratory expressed by nephrotic syndrome, accompanied by renal insufficiency is a rare occurrence in children; gentamicin and carbimazol can be criminalized. The suffering or impairment may be improved by removing the causative drug. Treatment failure was associated with duration of drug aggression and evolution of comorbidities.
\end{abstract}

Keywords: acute tubular necrosis, nephrotic syndrome, acute renal failure

Multiple therapies have renal side effects. The most common form of acute renal failure is acute tubular necrosis. There are rare occasions, seldom quoted, of tubular necrosis manifestating as nephrotic syndrome (1).

Case I is of an 5 months old infant, female, transferred from another health facility for generalized edema and nitrogen retention. With no significant pathological personal or family history until that time, the child was hospitalized for bronchopneumonia with fatal train under antibiotic treatment (12 days cephalosporin + Gentamicin). A day after discharge she presented with paleness, fever, vomiting, flatulence and generalized edema occu- rred after 2 days. She associated anemia and nitrogen retention, and she was transferred to our clinic. Clinical examination on admission revealed influenced general condition, with no fever, agitated, mucocutaneous pallor, generalized edema, oliguria $(0.23 \mathrm{~mL} / \mathrm{kg} / \mathrm{h})$,

Diagnosis of this stage involved:

- Hemolytic uremic syndrome, but the infant associated only normochromic, normocytic anemia with normal values of reticulocytes, platelets without schizocytes (PLT $=543,000$ / $\mathrm{mm}^{3}$ ).

- Acute pyelonephritis associated urinary tract disorders in infant - sterile urine cul- 


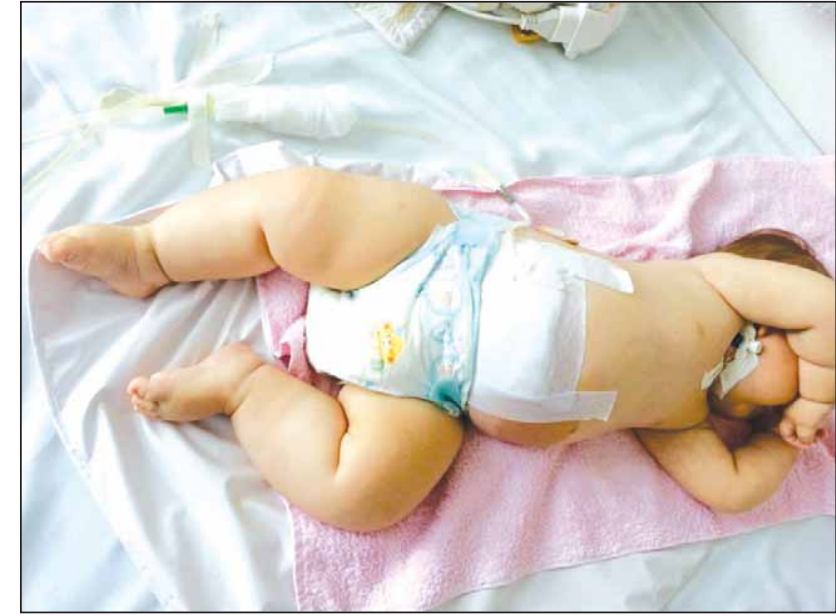

FIGURE 1. Generalized edema

ture and renal ultrasound excluded bladder or renal malformations.

- Nephrotic syndrome due to the associated dyslipidemia, marked hypoproteinemia, hematuria, proteinuria.

Testing negative for hepatitis $\mathrm{B}$ and $\mathrm{C}$ viruses ruled out secondary nephrotic syndrome. Given the young age we suspected congenital nephrotic syndrome, requiring confirmation by morphological evaluation.

Rough and rapid deterioration of kidney function $(\mathrm{BUN}=114 \mathrm{mg} / \mathrm{dl}$, creatinine $4.16 \mathrm{mg} / \mathrm{dl}$, hyperuricemia $-7.9 \mathrm{mg} / \mathrm{dl}$ ) to a GFR of $6.6 \mathrm{ml} / \mathrm{min} / 1.73 \mathrm{~m}^{2}$, and metabolic acidosis refractory to therapy, required placing a catheter for peritoneal dialysis. On that occasion it was performed and intraoperative renal biopsy. It highlighted the proximal renal tubules with degenerative changes granulocyte-type outbreaks of patchy necrosis, images of regeneration specific for drug-induced acute tubular necrosis.
The positive diagnosis was drug-induced acute tubular necrosis, acute renal failure, secondary nephrotic syndrome, secondary anemia. The treatment consisted in correcting electrolyte and acid-base disorders, correction of anemia with red blood cell transfusion, correcting hyperuricemia with allopurinol and extra-renal dialysis treatment for 10 which was well tolerated. The evolution was slow, with the resumption of diuresis and decreasing of nitrogen retention. Subsequent checks revealed the disappearance of clinical and biological picture of nephrotic syndrome and normalized renal parameters.

Case II is of a 15 years old teenager who presented with generalized swelling, sudden weight gain, appeared in a week. She was diagnosed in 2012 (age 13) at The National Institute of Endocrinology "C.I. Parhon", Bucharest, with Basedow disease, secondary amenorrhea. Under carbimazol treatment and intermittent with Euthyrox (for hypothyroidism associated episodes), she suddenly develops generalized edema. Physical exam reveals good general mood, $10.5 \mathrm{~kg}$ weight gain from the previous hospitalization (2 months earlier), exophtalmia, generalized edema. She was normotensive, and associated elastic, smooth, mobile, painless Grade I WHO goiter. Biological we confirmed typical appearance of impure nephrotic syndrome (inflammatory syndrome, hypoproteinemia $35.07 \mathrm{~g} / 1$, hypoalbuminemia, hypogammaglobulinemia, dyslipidemia with hypercholesterolemia $656 \mathrm{mg} / \mathrm{dL}$ and high triglycerides $474 \mathrm{mg} / \mathrm{dL}$, proteinuria $3 \mathrm{~g} / 1$, hematuria $3+$ ). We discussed secondary causes of nephrotic syndrome in teenagers and we evaluated HBsAg, HCV Ab, HIV, RPR negative. Tuberculin skin test was negative.
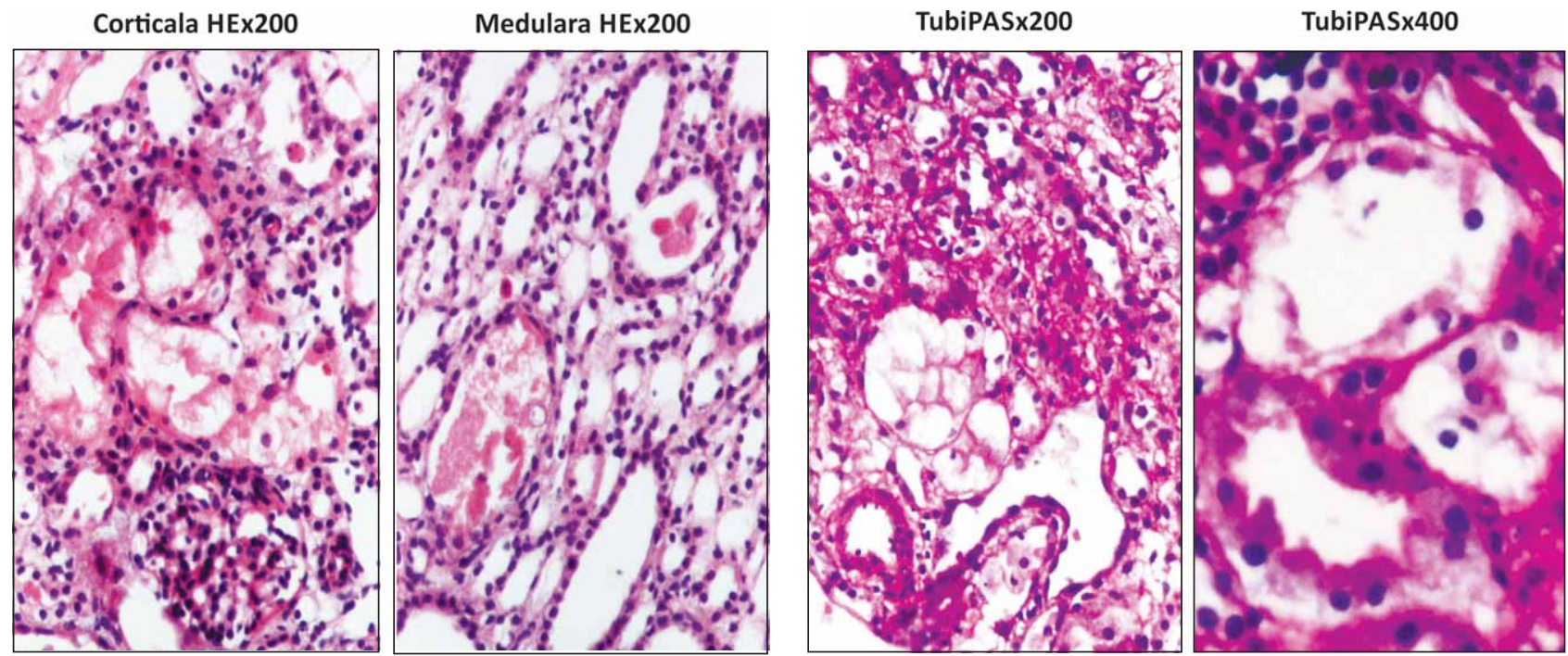

FIGURE 2. Degenerative changes granulocyte-type outbreaks of patchy necrosis 


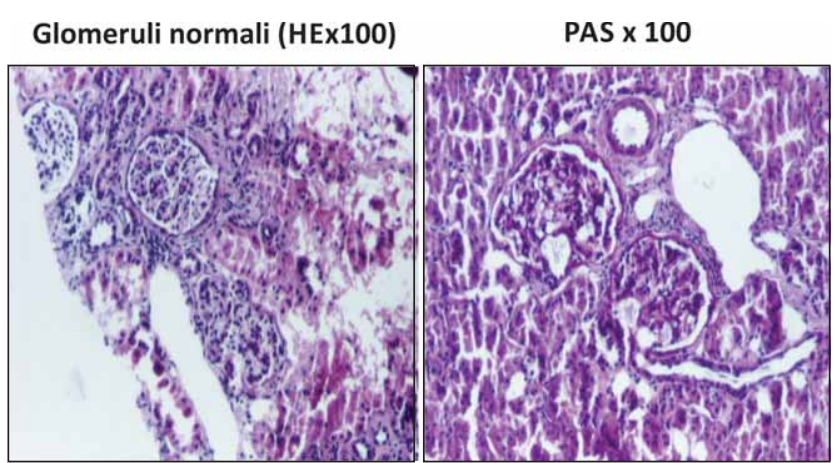

FIGURE 3. Normal glomerules, interstitial fibrosis, tubular necrosis

Normal AAN, lupus cells, ANA profile 9 and C3 ruled out a systemic disease. Thyroid parameters were within normal limits at that time. In that state we reviews the diagnosis of primitive impure nephrotic syndrome, Basedow disease. Imunosupressive therapy was initiated with the classical cortisone scheme, but it was unresponsive which imposed the Mendoza scheme. A percutaneous renal biopsy was performed to elucidate the morphological aspect. The immunofluorescence test revealed negative and the paraffin test showed minimum glomerular lesions, vacuolar tubular degeneration areas with outbreaks of cell necrosis with tubular atrophy and interstitial fibrosis. The conclusion was chronic active toxic tubular necrosis.

Simultaneously there has been decline in the renal function (GFR by Schwartz - $52 \mathrm{ml} / \mathrm{min} / 1,73$ $\mathrm{mp})$. It was necessary to cease the nephrotoxic antithyroid therapy and went into emergency thyroidectomy. Later, after a week of surgery, edema resolved, but kidney function continued to depreciate. The patient maintained dyslipidemia, proteinuria and hematuria, which required monitoring.

\section{RESULTS}

Both cases illustrate the renal tubular toxicity effect of the drugs used in the treatment of pediatric
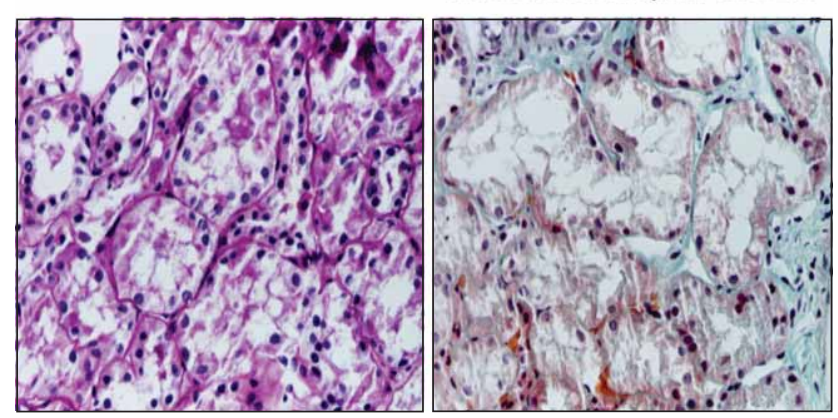

pathology - nonsteroidal anti-inflammatory (1), aminoglycosides (2), vancomycin (3), contrast media, gold salts. The peculiarity lies in clinical and biological expression of acute tubular necrosis as a nephrotic syndrome. In the first case where the toxic exposure was of short time, there has been full recovery of renal function compared to the second case where the outcome was interstitial fibrosis as toxic acted much longer. The literature quoted numerous cases of interstitial nephritis tubulointerstitial toxicity due to aminoglycoside therapy $(2,4)$, but only one case of interstitial nephritis after carbimazol treatment (72 years old male) (5). It is known so far the link between propylthiouracil and kidney damage.

\section{CONCLUSIONS}

It is known the nephrotoxic role of aminoglycosides. Carbimazol was only incriminated in some cases of acute tubular necrosis. In very rare cases a nephrotoxic trigger causes a nephrotic syndrome clinical and biological aspect.

\section{REFERENCES}

1. Jason M. Misurac, Chad A. Knoderer, Jeffrey D. Leiser and colab. Nonsteroidal Anti Inflammatory Drugs Are an Important Cause of Acute Kidney Injury in Children. The Journal of Pediatrics, June 2013, Vol. 162, 6:1153-1159.

2. Bălgradean Mihaela, Cinteză Eliza, Filipoiu F., Jinga V., Gentamicin, infections, and acute tubular necrosis in children. Farmacia, FARMACIA, 2013, Vol. 61, 4:772-780

3. McKamy S., Hernandez E., Jahng M., et al. Incidence and Risk Factors Influencing the Development of Vancomycin Nephrotoxicity in Children. The Journal of Pediatrics, 2011; Vol. 158, 3:422-426

4. Zappitelli M., Moffett J., Hyder A., Goldstein S. Acute kidney injury in non-critically ill children treated with aminoglycoside antibiotics in a tertiary healthcare centre: a retrospective cohort study. Nephrol. Dial. Transplant, 2011. 26 (1): 144-150

5. Day C., Bridger J., Rylance P., et al. Leukocytoclastic vasculitis and interstitial nephritis with carbimazole treatment. Nephrol Dial Transplant 2003; 18: 429-431 